\title{
Fundamental design of a distributed erbium-doped fiber amplifier for long-distance transmission
}

Rottwitt, Karsten; Bjarklev, Anders Overgaard; Povlsen, Jørn Hedegaard; Lumholt, Ole; Rasmussen, Thomas $\mathbf{P}$

Published in:

Journal of Lightwave Technology

Link to article, DOI:

$10.1109 / 50.184892$

Publication date:

1992

Document Version

Publisher's PDF, also known as Version of record

Link back to DTU Orbit

Citation (APA):

Rottwitt, K., Bjarklev, A. O., Povlsen, J. H., Lumholt, O., \& Rasmussen, T. P. (1992). Fundamental design of a distributed erbium-doped fiber amplifier for long-distance transmission. Journal of Lightwave Technology, 10(11), 1544-1552. https://doi.org/10.1109/50.184892

\section{General rights}

Copyright and moral rights for the publications made accessible in the public portal are retained by the authors and/or other copyright owners and it is a condition of accessing publications that users recognise and abide by the legal requirements associated with these rights.

- Users may download and print one copy of any publication from the public portal for the purpose of private study or research.

- You may not further distribute the material or use it for any profit-making activity or commercial gain

- You may freely distribute the URL identifying the publication in the public portal 


\title{
Fundamental Design of a Distributed Erbium-Doped Fiber Amplifier for Long-Distance Transmission
}

\author{
Karsten Rottwitt, Anders Bjarklev, Jørn Hedegaard Povlsen, Ole Lumholt, and Thomas P. Rasmussen
}

\begin{abstract}
Comprehensive theoretical analysis on the design of a distributed erbium doped fiber amplifier for long-distance transmission has been carried out, using a highly accurate model. The dispersion of the optical fiber as a function of the numerical aperture and the cutoff wavelength is included. Designs based on a bidirectional pumping scheme are evaluated, taking nonlinearities into account. The optimum value of the numerical aperture will be evaluated for cutoff wavelengths where the propagating pumppower is single moded. For distances between each pumping station in the region between 10 and $100 \mathrm{~km}$, the optimum ratio of co- and counterpropagating pump power will also be evaluated.
\end{abstract}

\section{INTRODUCTION}

$\mathbf{R}$ ECENTLY distributed erbium-doped fiber amplifiers (EDFA's) have attracted large attention, both for bus networks with uniformly distributed gain [1], and as a promising transmission medium for high bitrate optical pulses over ultra-long distances [2]. For both applications it is the low generation of amplified spontaneous emission (ASE) that is the attractive property. The ability of limiting the energy fluctuation along the transmission line is advantageous through the use of distributed amplifiers compared to lumped gain systems. The focus of designing distributed EDFA's is therefore different from achieving gain-coefficient effective active fibers for lumped gain amplifiers, thus we will show the special aspects of optimization of distributed EDFA's. The optimum fiber design parameters will be demonstrated, taking into account that the fiber dispersion varies with the numerical aperture of the optical fiber. Special attention is given to the question of how to choose the best ratio between co- and counterpropagating pump power.

The analysis is based on an experimentally verified and highly accurate model for the EDFA [3], which takes the detailed spectrum of forward and backward travelling ASE into account. Model inputs are the experimentally determined wavelength dependent absorption and emission cross sections for a $\mathrm{Ge} / \mathrm{Er}$ fiber [4] as well as the intrinsic loss. Considering the pump wavelength, it has been demonstrated that the $1.48-\mu \mathrm{m}$ pump wavelength for the distributed EDFA is more efficient compared to the $0.98-\mu \mathrm{m}$ pumping band, due to the low intrinsic losses at $1.48 \mu \mathrm{m}$, even though this results in increased noise [5]. For all the following analysis we have

Manuscript received November 8, 1991; revised June 9, 1992. This was supported in part by the Danish Technical Research Council and by the National Agency of Industry and Trade, Denmark.

The authors are with the Center for Broadband Telecommunications, Electromagnetics Institute Technical University of Denmark, DK-2800 Lyngby, Denmark.

IEEE Log Number 9203432 chosen to use this pumping band, and a fixed pump power of $100 \mathrm{~mW}$. Furthermore we have used a signal wavelength equal to $1.535 \mu \mathrm{m}$, where the EDFA has a peak in its emission cross-section spectrum.

\section{THEORY}

For transmission systems based on lumped gain taking the nonlinear Kerr effect into account the dispersion is an essential parameter. For ultra-long transmission it is the mean dispersion of the transmission line that is important when the repeater length is shorter than the dispersion length. The dispersion length is the distance where the pulsewidth is $2^{1 / 2}$ times the initial pulsewidth, when the nonlinear Kerr effect is neglected. This length is given by the fiber dispersion and the pulsewidth of the optical pulse [6]. Therefore in a system employing lumped gain the amplifier dispersion may be negative without influencing the system performance, as long as the mean dispersion is positive, when the nonlinear Kerr effect is used to form optical solitary pulses. In a transmission system based on distributed gain the mean dispersion is equal to the dispersion of one distributed EDFA. Therefore it is essential to look at the dispersion of each distributed EDFA. As a first-order approximation we assume that the dispersion of the fiber is calculated using the usual theory for passive fibers and neglecting the influence on the refractive-index profile from the nonlinear refractive index. The total fiber dispersion is computed using an accurate model for propagation of electrical fields in an optical fiber [7]. The effective core area which is essential for the pulse power and thus the timing jitter [8], are evaluated from the same model, by use of the relation

$$
A_{\mathrm{eff}}=\frac{\left(\int_{A} \psi^{2}(r, \theta) d A\right)^{2}}{\int_{A} \psi^{4}(r, \theta) d A}
$$

$\psi(r, \theta)$ is the modal distribution of the fundamental fiber modefield and $A$ is the cross section of the fiber, where $(r, \theta)$ are polar coordinates [9]. The $A_{\text {eff }}$ is only dependent on the index profile of the fiber, that is the numerical aperture and the coreradius, as this defines the modal field distribution. Thus the $A_{\mathrm{eff}}$ is calculated without influence of any of the design parameters concerning the erbium dopant profile. For an ideal transmission line the gain coefficient is at a constant level which is equal to one. In a real transmission line either based on lumped gain or distributed gain, the gain varies along the transmission line, and ASE power is accumulated. The gain variation along the transmission line is described by the relative gain, $\gamma(x)$, and results in pulse power fluctuations as a 
function of the length coordinate $x$. The fidelity of a distributed fiber is evaluated on the basis of the main errors, which affect the transmission system. These are fluctuations of the detected pulse power and uncertainty in the pulse arrival times [10].

For ultra-long pulse transmission over several distributed amplifiers, we define the distance between two pumping stations as the amplification period. When the total transmission length is much larger than the distance of one amplification period, the signal to noise ratio after each distributed EDFA is calculated according to values for the signal and noise power levels after the amplifier. For distributed amplification two boundary cases exist, copropagating pump and signal and counterpropagating pump and signal. Between these cases, the fraction of the two pumppowers may be varied continuously. Considering the case of only copropagating pump and signal the power level of both signal and noise is increased in the beginning of the amplification period and attenuated in the end of the amplification period. Therefore the mean power level along the transmission line is higher than the initial power level for both the signal and noise power. For the counter propagating pump and signal the situation is reversed. In this situation the power level of both signal and noise is attenuated in the beginning of the amplification period and increased in the end of the amplification period. Hence, in this situation the power level is lower than the initial power level for both the signal and noise power. In order to take advantage of the nonlinear Kerr effect to form solitary pulses, the mean energy along the transmission line in a signal given by a first-order soliton is [6]

$$
\langle E\rangle=\ln (\sqrt{2}+1) \cdot \frac{D}{\tau} \cdot \frac{\lambda^{3} \cdot A_{\mathrm{eff}}}{\pi^{2} \cdot c \cdot n_{\mathrm{ke}}}
$$

$D$ is the fiber dispersion, $\tau$ the pulsewidth at half maximum intensity, $\lambda$ is the signal wavelength, $A_{\text {eff }}$ is the effective core area, $c$ is the velocity of the light, and $n_{\mathrm{ke}}$ is the nonlinear Kerr coefficient. Assuming that the dispersion length is much larger than the amplification period the power level for both signal and noise at point $z$ along the transmission line is expressed as in [6]

$$
P(z)=P_{0} \cdot \exp \left(\int_{0}^{z} \gamma(x) d x\right)
$$

For distributed EDFA's it must be noted that

$$
\exp \int_{0}^{L} \gamma(x) d x=1 \text {. }
$$

Where $L$ is the amplification period. From (3) we see that the path average power is given by

$$
\langle P\rangle=P_{0} \cdot \frac{1}{L} \cdot \int_{0}^{L} \exp \left(\int_{0}^{z} \gamma(x) d x\right) d z=P_{0} \cdot R_{g}
$$

From this

$$
\langle E\rangle=E_{s} \cdot R_{g} .
$$

Where $E_{s}$ is the signal energy in the beginning of the amplification period. Considering the distributed amplifier as a discrete amplifier in the overall transmission line the noise energy in the signal mode, after each amplification period is

$$
\tilde{E}=\tilde{n} \cdot h \cdot \nu_{s}
$$

where $\tilde{n}$ is the number of photons in the signalmode generated by amplified spontaneous emission, and $h \cdot \nu_{s}$ is the photon energy at the signal frequency $\nu_{s}$. After $N=L_{\text {tot }} / L$ numbers of amplification periods the noise energy in the signal mode is

$$
\tilde{E}=L_{\text {tot }} \cdot \alpha_{\text {ASE }} \cdot h \cdot \nu_{s} / 2
$$

where $\alpha_{\mathrm{ASE}}$ is related to the noise figure through $\alpha_{\mathrm{ASE}}=$ $2 \tilde{n} / L=(F-1) / L$. The factor of 2 arises as the generated ASE photons are equally divided in the two orthogonal polarizations of the fiber. Assuming that the detector consists of an optical filter of bandwidth $B_{0}$ followed by a fotodetector and an integrator the energy within one timeslot $\Delta T$ is measured [10]. The number of communication channels of which the receiver is sensitive $M$ is then $M=K \cdot B_{0} \cdot \Delta T$, where $K=1$ if the detector is sensitive to one polarization and $K=2$ if the detector is sensitive to two polarizations. In Appendix A we show that the electrical signal to noise ratio for a mark in a digital signal is

$$
\frac{S}{N}=\frac{E_{s}+2 M \tilde{E}}{2 \tilde{E}}=\frac{\langle E(1)\rangle+2 M \tilde{E} \cdot R_{g}}{2 \tilde{E} \cdot R_{g}}
$$

where $E_{s}$ is the signal energy in a mark in the beginning of an amplification period defined by (6). From the above description evidently the product $\alpha_{\mathrm{ASE}} \cdot R_{g}$ is an essential figure. The distributed amplifier is optimally designed with respect to influence of the noise, by minimizing the product $\alpha_{\mathrm{ASE}} \cdot R_{g}$. The factor $\alpha_{\mathrm{ASE}}$ is as mentioned the number of generated ASE photons pr. Hertz, pr. second and pr. unit length of the amplifier.

The mean number of photons $\langle n\rangle$ along the transmission line is related to the material parameters through the following differential equation as shown in Appendix A

$$
\frac{d\langle n\rangle}{d z}=g \cdot\langle n\rangle+\alpha_{e}
$$

where $g=\alpha_{e}-\alpha_{a}-\alpha_{i}$ [12]. $\alpha_{e}$ and $\alpha_{a}$ are the emission and absorption factors determined from the emission and absorption cross sections, respectively [12], $\alpha_{i}$ is the normal fiber attenuation constant, representing absorption. For an ideal transmission line $g=0$, that is $\alpha_{e}=\alpha_{a}+\alpha_{i}$, in the case of fully inversion furthermore $\alpha_{a}=0$. Pumping at $1.48 \mu \mathrm{m}$ fully inversion is impossible. Assuming that the emission and absorption cross section are equal at the signal wavelength $1.535 \mu \mathrm{m}$, and that $70 \%$ of fully inversion is achieved we obtain $\alpha_{e}=\alpha_{i} \cdot 1.74$. With this (10) results in the equation

$$
\langle n\rangle=\alpha_{e} \cdot L
$$

where $L$ is the length of the considered fiber. In this ideal case the generated number of ASE photons pr. Hertz, pr. second and pr. unit length are equal to $\alpha_{i} \cdot 1.74$. This implies the inequality

$$
\alpha_{\mathrm{ASE}}>1.74 \cdot \alpha_{i}
$$




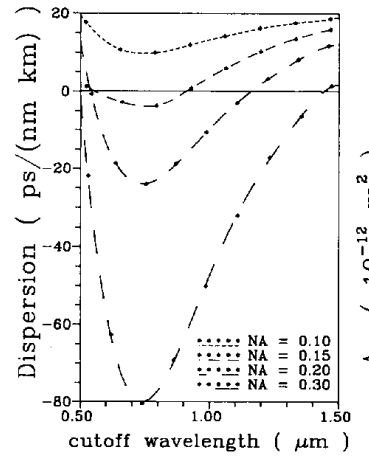

(a)

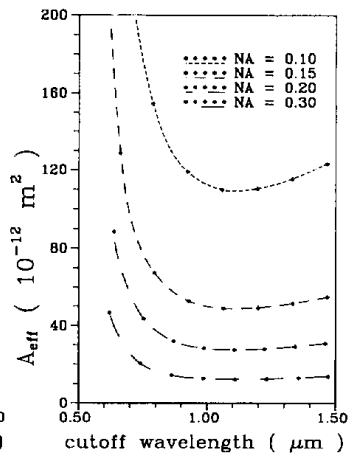

(b)
Fig. 1. (a) The total fiber dispersion and (b) the effective core area, both calculated for a step index profile as a function of the LP $P_{11}$ cutoff wavelength with the numerical aperture of the fiber as parameter.

For a fiber with $0.23 \mathrm{~dB} / \mathrm{km}$ loss $\alpha_{i}$ is equal to 52.96 . $10^{-3} \mathrm{~km}^{-1}$, and thus $\alpha_{i} \cdot 1.74=92.31 \cdot 10^{-3} \mathrm{~cm}^{-1}$ equal to $0.4 \mathrm{~dB} / \mathrm{km}$.

Besides the noise power error, another major error source is timing jitter. This error occurs when spectral noise is added to the signal, resulting in fluctuations of the group velocity of a transmitted pulse train, which results in timing jitter, often denoted as the Gordon Haus effect [8]. In Appendix B we derive an expression for the variance for the arrival time of a pulse $\left\langle(\partial t)^{2}\right\rangle$ in accordance to the above theory for noise and energy fluctuation. In agreement with [10] the variance is calculated to be

$$
\left\langle(\delta t)^{2}\right\rangle=\frac{1.763 \cdot h}{9} \cdot n_{\mathrm{ke}} \cdot \frac{D \cdot L_{\mathrm{tot}}^{3}}{\tau \cdot A_{\mathrm{eff}}} \cdot \frac{\alpha_{\mathrm{ASE}}}{2} \cdot R_{g} .
$$

In Appendix B we have shown that the timing jitter reduces with increasing signal power and decreasing noise power. Equation (2) shows that the higher $A_{\text {eff }}$ the higher signal power level is required to support soliton operation. Thus from this point of view fibers with high $A_{\text {eff }}$ are desirable. An increasing $A_{\text {eff }}$ may be achieved by reducing the cutoff wavelength for a fixed NA according to Fig. 1(b). However this results in a reduced dispersion that for NA higher than 0.15 is negative. For soliton operation a positive mean dispersion is necessary for transmission of optical solitary pulses [6]. In lumped gain systems the mean dispersion is the average of the dispersion in the passive fiber section and the following active fiber section. Therefore in lumped gain systems the restrictions to NA, cutoff wavelength and $A_{\text {eff }}$ are not as important as in distributed active fibers. Thus we emphasize that timing jitter is reduced by reducing the noise power relative to the signal power level, but in a transmission line based on distributed amplification at the same time it is a necessity to assure a positive mean dispersion.

\section{RESULTS}

Computing the dispersion for a fiber with a step-index profile as a function of the cutoff wavelength for the LP $_{11}$ mode and with the numerical aperture (NA) as parameter the results in Fig. 1(a) are achieved. Fig. 1(b) shows the effective core area, for the same refractive-index profiles, calculated by integration of the electrical field in the fiber, as described earlier. From Fig. 1(a) it is clear that NA has to be kept below 0.3 to assure positive dispersion, when the cutoff wavelength has to be low enough to ensure that the fiber is operated with a single pump mode. For NA equal to 0.2 the dispersion change from positive to negative values for cutoff wavelengths lower than $1.16 \mu \mathrm{m}$. Having NA equal to 0.1 the dispersion is positive for any cutoff wavelength. The curves show a minimum dispersion between 700 and $800 \mathrm{~nm}$ where the waveguide dispersion is most pronounced. For any fixed value of the numerical aperture the total fiber dispersion is dominated by the material dispersion for small values of the cutoff wavelength, as the electrical field in this situation is poor guided. For large values of the cutoff wavelength the electrical field is broadened, hence again the total fiber dispersion is dominated by the material dispersion. In the region between these two boundary cases the waveguide dispersion is influencing and therefore an extremum is formed.

The effective core area decreases with increasing numerical aperture. This is due to the guiding properties of the high refractive index obtained in the fiber core, the higher index difference and hence the higher numerical aperture, the better guiding property and therefore the lower effective core area. For the various values of cutoff wavelength, there exist a minimum effective core area near cutoff wavelengths equal to $1 \mu \mathrm{m}$. For small cutoff wavelengths, the electrical field is poor guided, hence a large $A_{\text {eff }}$. For larger values of the cutoff wavelengths, the electrical field becomes well guided and therefore $A_{\text {eff }}$ is decreased. For even larger cutoff wavelengths the electrical field is broadened and therefore $A_{\text {eff }}$ is increased.

As described previously the product $\alpha_{\mathrm{ASE}} \cdot R_{g}$ is an important figure, when the two system errors, noise power error and timing jitter, are considered. Calculation of this product with the numerical aperture and the cutoff wavelength as parameter gives the results shown in Fig. 2. This figure is calculated for a $100-\mathrm{km}$-long distributed EDFA with equal backward and forward propagating pump. The confinement factor, defined as the erbium dopant radius relative to the fiber core radius, is equal to 0.8 . Even though these values have been fixed, further calculations have shown that Fig. 2 is qualitative valid for other values of the above parameters. The solid curve represents the zero dispersion limit. Above this limit the total fiber dispersion is positive, where it is negative below. The figure clearly demonstrates that the optimum fiber is achieved, using as high numerical aperture as possible, but at the same time in the region where the total fiber dispersion is positive. An important issue is however that the background loss increases with increasing NA due to the introduced stress, that occurs when the refractive index difference between core and cladding is large. Further calculations have shown that both the necessary pump power for transparency and the noise figure, and therefore also $\alpha_{\mathrm{ASE}}$ increases with increasing background loss. For a pump power of $100 \mathrm{~mW}$ it is found that the noise figure changes from 13 to $14 \mathrm{~dB}$ when the background loss is changed from 0.23 to $0.26 \mathrm{~dB} / \mathrm{km}$ for a signal wavelength of $1.554 \mu \mathrm{m}$ [15]. 


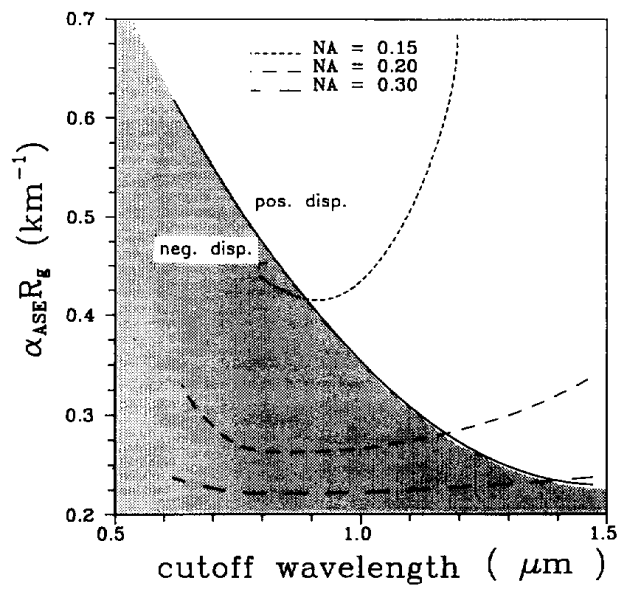

Fig. 2. The $\alpha_{\mathrm{ASE}} \cdot R_{g}$ product as a function of the $\mathrm{LP}_{11}$ cutoff wavelength for different values of the numerical aperture. The total fiber dispersion is negative in the gray area.

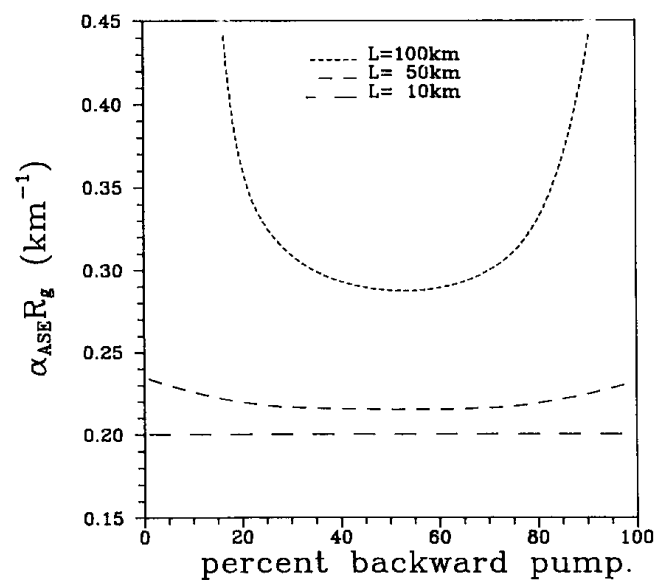

Fig. 3. The $\alpha_{\mathrm{ASE}} \cdot R_{g}$ product as a function of the backward propagating pumppower relative to the forward propagating pumppower, for three different fiberlengths.

In a long transmission line consisting of cascade coupled distributed EDFA's it is as mentioned earlier essential to maintain a small and positive dispersion for propagation of solitary pulses. Therefore we have for the following discussion chosen to use a fiber with the numerical aperture equal to 0.2 and the cutoff wavelength equal to $1.22 \mu \mathrm{m}$ and a backgroundloss of $0.23 \mathrm{~dB} / \mathrm{km}$. According to Fig. 1(a) and (b) this gives a total dispersion equal to $3.2 \mathrm{ps} /(\mathrm{nm} \cdot \mathrm{km})$ and an effective core area equal to $27.7(\mu \mathrm{m})^{2}$. The total ASE power copropagating with the signal was in all the simulations in the order of magnitude of $10^{-6} \mathrm{~W}$ which is far from the saturation level. This fiber gives as shown in Fig. 2 an $\alpha_{\mathrm{ASE}} \cdot R_{g}$ product equal to $0.29 \mathrm{~km}^{-1}$ for $50-\mathrm{mW}$ pump power copropagating as well as counterpropagating with the signal. Fig. 3 shows the $\alpha_{\mathrm{ASE}} \cdot R_{g}$ product as a function of the ratio between forward and backward propagating pump, for different lengths of the distributed EDFA. From (5) and (12) it is seen that the product $\alpha_{\mathrm{ASE}} \cdot R_{g}$ is different using backward and forward pumping respectively and lowest for the shortest fiber.

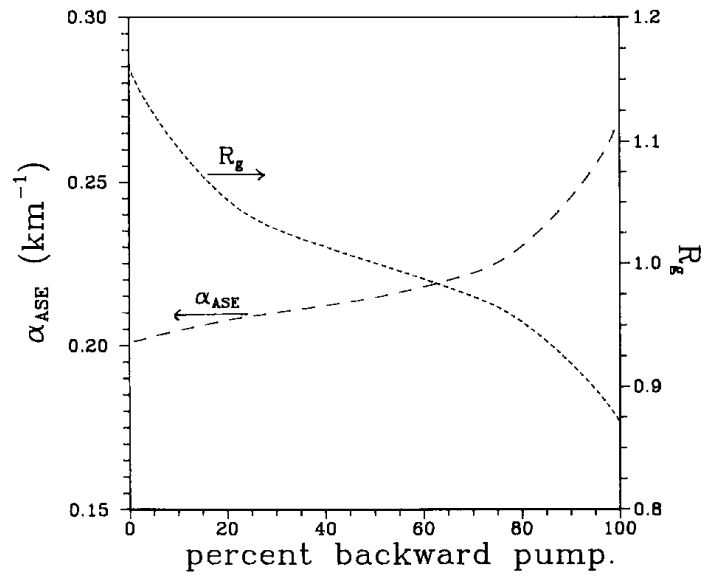

Fig. 4. $\alpha_{\text {ASE }}$ and $R_{g}$ as functions of the backward propagating pumppowe relative to the forward propagating pumppower in a $50-\mathrm{km}$-long fiber with $\mathrm{NA}=0.2$ and cutoff wavelength equal to $1.22 \mu \mathrm{m}$.

Generally it is obvious that the lowest $\alpha_{\mathrm{ASE}} \cdot R_{g}$ product is achieved for the shortest length of fiber, it is also worth noting that the curves show a minimum near an equal ratio between backward and forward propagating pump. The precise minimum is located for backward propagating pumppowers a little higher than the forward propagating pumppower. The displacement from an equal ratio is though negligible, therefore we conclude that the optimum pumping scheme is equally pumping of the fiber from both ends. The curves are not symmetric, they show an advantage in $100 \%$ counterpropagating pump and signal compared to $100 \%$ copropagating pump and signal. This is explained by the two factors represented in the $\alpha_{\mathrm{ASE}} \cdot R_{g}$ product. The coefficient $R_{g}$ which expresses the mean power level deviation from the initial power level is larger than one for the copropagating pump and less than one for the counterpropagating pump. This is shown in Fig. 4 , which shows the factors $R_{g}$ and $\alpha_{\text {ASE }}$ as a function of the ratio between forward and backward propagating pump. The used fiberlength for this figure is $50 \mathrm{~km}$, with a numerical aperture for the fiber equal to 0.2 and a cutoff wavelength equal to $1.22 \mu \mathrm{m}$. The curve is not symmetric around $R_{g}=1$, which is due to the accumulated relative gain, that is described by an exponentially relation. The accumulated relative gain is a function that begins and ends at zero, but is positive for the copropagating pumping scheme, and negative for the counterpropagating pump. Averaging the accumulated gain over one amplification period, as in (5), which has the accumulated relative gain as argument results in a factor $R_{g}$ greater than one for copropagating pump and less than one for the counter propagating pump. The deviation from one is largest in the copropagating pumping scheme, as the argument to the accumulated gain is a positive function along the distributed EDFA.

The coefficient $\alpha_{\text {ASE }}$, which is the number of generated ASE photons pr. unit length, is also shown in Fig. 4. $\alpha_{\mathrm{ASE}}$ has the lowest values in the copropagating pumping scheme. This is explained considering the distributed fiber as a series of discrete amplifiers. The noise figure for each discrete 
amplifier is $F_{n}=\left(n_{n}+1\right) / G_{n}$, where $n_{n}$ is the number of ASE photons generated in the $n$ 'th amplifier which has gain $G_{n}$. Considering a distributed fiber having backward propagating pump, the gain in a discrete fiber $G_{n}$ figurative in the beginning of the distributed fiber is less than one, therefore the noise figure is determined by $G_{n}$. For a copropagating pumping scheme the gain of a discrete fiber in the beginning of the distributed fiber is larger than one. The noise figure is therefore determined by both $n_{n}$ and $G_{n}$, and hereby less than in the counterpropagating situation. Looking at the total distributed fiber the overall noise figure is higher than a noise figure in any infinitesimal part of the fiber. The noise in a forward pumped distributed fiber is therefore lower than in a backward pumped distributed fiber. Fundamentally $\alpha_{\mathrm{ASE}}$ is above the limit given by $\alpha_{i} \cdot 1.74=92.31 \cdot 10^{-3} \mathrm{~km}^{-1}$ as mentioned previously.

The performance of an ultra long transmission line may be evaluated according to (9) and (13) for the noise power error as well as the timing jitter both represented through bit error rates (BER's). As an example of a 9000-km-long transmission system based on distributed amplification without any ASE or timing jitter reducing components, we consider two systems both operating with a cascade coupling of 90 similar fibers. We have chosen the fiber used in Fig. 3, having a numerical aperture equal to 0.2 and a cutoff wavelength equal to $1.22 \mu \mathrm{m}$. The dispersion is $3.2 \mathrm{ps} /(\mathrm{nm} \cdot \mathrm{km})$ and the effective core area is $27.7(\mu \mathrm{m})^{2}$. The mean signal power level in the calculations is $0.1 \mathrm{~mW}$, and the signal wavelength is equal to $1.535 \mu \mathrm{m}$. System one operates with a timeslot $1 / B=5 \cdot \tau$, where $B$ is the bitrate and $\tau$ the pulse width at half maximum intensity, and system two operates with a timeslot $1 / B=10 \cdot \tau$. A fixed signal power level result in the bitrates 2.6 and $1.8 \mathrm{~Gb} / \mathrm{s}$, respectively. The chosen parameters for the two systems assure that the amplification length is much smaller than the dispersion length, in order to assure stable soliton transmission [14]. The noise power error ratio is transformed to BER by assuming Gaussian distribution of the detected energy as specified in Appendix A. The mean and variance of the total energy in a bitperiod for a space are both equal to $M$ in (9) as shown in Appendix A. For a mark the mean and variance of the signal energy relative to the noise energy $S_{1}$, are $S_{1}+M$ and $2 S_{1}$ respectively, where $S_{1}$ is calculated through (9) as described in Appendix A. BER from timing jitter is calculated assuming that the arrival time of a pulse follows a Gaussian distribution where the variance is given in (13). Fig. 5(a) and (b) shows BER's from timing jitter and the noise power error versus the product of $\alpha_{\mathrm{ASE}}$ and $R_{g}$. For the noise power error BER's are shown for three different values of $M$. We conclude from this figures that for both the 1.8-Gb/s system in Fig. 5(a) and the $2.6-\mathrm{Gb} / \mathrm{s}$ system in Fig. 5(b) the BER is limited by timing jitter for any value of $\alpha_{\mathrm{ASE}} \cdot R_{g}$. This implies that the timing jitter is the essential parameter, and for BER less than $10^{-12}$ we have to ensure that $\alpha_{\mathrm{ASE}} \cdot R_{g}$ is less than 0.3 for the $2.6-\mathrm{Gb} / \mathrm{s}$ system and less than 0.45 for the $1.8-\mathrm{Gb} / \mathrm{s}$ system. Looking at Fig. 2 and Fig. 3 we see that $\alpha_{\mathrm{ASE}} \cdot R_{g}$ may be reduced either by using a fiber with a high NA and hence a high cutoff wavelength or by reducing the spacing between two

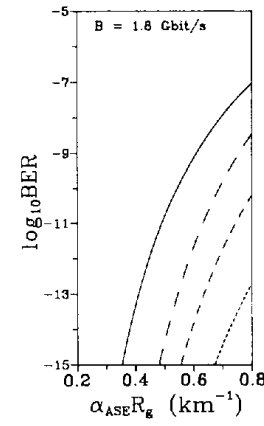

(a)

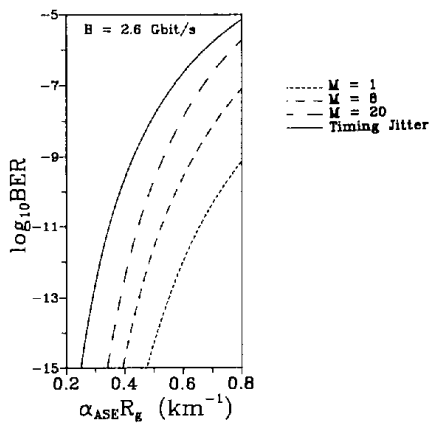

(b)
Fig. 5. The BER for (a) a $1.8-\mathrm{Gb} / \mathrm{s}$ and (b) a $2.6-\mathrm{Gb} / \mathrm{s}$ system, with the $\alpha_{\mathrm{ASE}} \cdot R_{g}$ product as parameter.

pumpstations. The two systems we have investigated had an $\alpha_{\text {ASE }} \cdot R_{g}$ product equal to 0.3 for $100 \mathrm{~km}$ spacing between each pumpstation and equal to 0.2 for $10-\mathrm{km}$ spacing. Fig. 2 shows that $\alpha_{\mathrm{ASE}} \cdot R_{g}$ may be reduced to lower than 0.22 for $\mathrm{NA}=0.3$ and a spacing between the pumpstations between 50 and $100 \mathrm{~km}$. It is though very important to notice, that the above examples have a ratio $\tau / D$ equal to $24(\mathrm{~nm} \cdot \mathrm{km})$ and $17(\mathrm{~nm} \cdot \mathrm{km})$. In [10] it is shown, for lumped gain systems, that the timing jitter is the limiting phenomena for both these ratios. In future work it is therefore important to investigate the fraction $\tau / D$ for distributed fibers in ultra-long transmission lines.

\section{CONCLUSION}

Different design parameters and $1.48-\mu \mathrm{m}$ pumping schemes for a distributed EDFA are investigated. The evaluation is performed by inclusion of noise properties. From our analysis we conclude that the optimum distributed EDFA is achieved using an index profile with as large index difference as possible. An optimum value of the numerical aperture exists where the cutoff wavelength is adjusted to give a positive total fiber dispersion. For cutoff wavelengths where the pumppower is single moded the optimum numerical aperture is close to 0.3 with the cutoff wavelength in the range from 1.45 to $1.48 \mu \mathrm{m}$. The optimum pumping scheme is bidirectional pumping with equal co- and counterpropagating pump. Using a distributed EDFA with only one pump, the counterpropagating pump configuration is most advantageous. The ASE level in all our analysis is found to be below saturation level of the EDFA even after $9000-\mathrm{km}$ transmission. As a system example a $9000-\mathrm{km}$-long transmission line is studied using a fiber with NA equal to 0.2 , a cutoff wavelength equal to $1.22 \mu \mathrm{m}$, which results in a total fiber dispersion $D=$ $3.2 \mathrm{ps} /(\mathrm{nm} \cdot \mathrm{km})$ and an effective core area equal to 27.7 $(\mu \mathrm{m})^{2}$ at the signal wavelength equal to $1.535 \mu \mathrm{m}$. Timing jitter is found to be the most critical noise contribution. However $2.6 \mathrm{~Gb} / \mathrm{s}$ error free transmission over $9000 \mathrm{~km}$ is though simulated with a spacing between two pumping stations equals to $100 \mathrm{~km}$. 


\section{APPENDIX A}

DERIVATION OF (9) FOR THE SIgNAL TO NOISE Ratio of a Mark IN a Digital SySTEM

Both the signal and the noise energy varies along the transmission line. Thus we emphasize that in this appendix it is the energy in the beginning of an amplification period that has to be inserted in all expressions which include energy terms. From [11] we adopt (A.1a) and (A.1b) for the mean number of photons pr. mode (photons pr. sec. pr. Hz.) propagating in the direction $z$. The total number of photons at the receiver are described by an addition of $n_{s}$ photons emitted from the signal source propagating in the signal mode and $\tilde{n}$ photons emitted due to spontaneous emission (ASE) propagating in the signal mode and $M-1$ other modes when the receiver is sensitive to $M$ modes.

$$
\begin{aligned}
\frac{d\langle\tilde{n}\rangle}{d z} & =\langle\tilde{n}\rangle\left(\gamma_{e}-\gamma_{a}-\alpha_{i}\right)+\gamma_{e} \\
\frac{d\left\langle n_{s}\right\rangle}{d z} & =\left\langle n_{s}\right\rangle\left(\gamma_{e}-\gamma_{a}-\alpha_{i}\right)
\end{aligned}
$$

where $\gamma_{e}$ and $\gamma_{a}$ are the emission and absorption factors of the erbium doped fiber [12] and $\alpha_{i}$ the intrinsic attenuation constant. $\langle\tilde{n}\rangle$ is the mean number of noise photons in one mode and $\langle n\rangle$ is the mean number of signal photons in the signal mode. Equation (A.1a) is valid for the ASE photons in $M$ modes, whereas (A.1b) only is valid for the signal photons. From (A.1b) the gain is calculated as

$$
G=\exp \left(\int_{z^{\prime}=0}^{z}\left(\gamma_{e}-\gamma_{a}-\alpha_{i}\right)\right) d z^{\prime}
$$

The second moment of the ASE photon number propagating in the $M-1$ modes is given in (A.3a), whereas the second moment of the signal and ASE photons propagating in the signal mode is given by (A.3b)

$$
\begin{aligned}
\frac{d\left\langle\tilde{n}^{2}\right\rangle}{d z}=2\left\langle\tilde{n}^{2}\right\rangle\left(\gamma_{e}-\gamma_{a}-\alpha_{i}\right)+\langle\tilde{n}\rangle\left(3 \gamma_{e}+\gamma_{a}+\alpha_{i}\right)+\gamma_{e} \\
\\
\begin{aligned}
\frac{d\left\langle\left(n_{s}+\tilde{n}^{2}\right)\right\rangle}{d z}= & 2\left\langle\left(n_{s}+\tilde{n}\right)^{2}\right\rangle\left(\gamma_{e}-\gamma_{a}-\alpha_{i}\right) \\
& +\left\langle\left(n_{s}+\tilde{n}\right)^{2}\right\rangle\left(3 \gamma_{e}+\gamma_{a}+\alpha_{i}\right)+\gamma_{e}
\end{aligned}
\end{aligned}
$$

Combining (A.1a) and (A.1b) with (A.3a) and (A.3b), respectively, $M$ equations for the variance of the photon number $v^{2}=\left\langle n^{2}\right\rangle-\langle n\rangle^{2}$ are achieved, all with the same form as (A.4).

$$
\frac{d v}{d z}=2\langle v\rangle\left(\gamma_{e}-\gamma_{a}-\alpha_{i}\right)+\langle n\rangle\left(\gamma_{e}+\gamma_{a}+\alpha_{i}\right)+\gamma_{e}
$$

Thus in (A.4) $n=n_{s}+\tilde{n}$ in the signal mode and in the $M-1$ other modes $n=\tilde{n}$. In the signal mode (A.4) have the boundary conditions $\left.\langle n\rangle\right|_{z=0}=n_{0}$ and $v_{z=0}=n_{0}$, as we assume that the signal source is coherent. In the $M-1$ other modes the boundary conditions are $\left.\langle n\rangle\right|_{z=0}=0$ and $\left.v\right|_{z=0}=0$. A general solution to (A.4) may be written in the form

$$
h(n)=n^{2}+n .
$$

Inserting (A.5) into (A.4) gives

$$
\frac{d v}{d z}-\frac{d h}{d z}=2(v-h)\left(\gamma_{e}-\gamma_{a}-\alpha_{i}\right) .
$$

Hence

$$
(v-h)=\left.(v-h)\right|_{z=0} \exp \left(\int_{z^{\prime}=0}^{z} 2\left(\gamma_{e}-\gamma_{a}-\alpha_{i}\right)\right) d z^{\prime} .
$$

Applying (A.2), (A.7) reduces to $(v-h)=\left.(v-h)\right|_{z=0} \cdot G^{2}$. Using the boundary conditions, the variance at the point of detection $z$ in the signal mode is given by

$$
v_{0}=\left(n_{s}+\tilde{n}\right)^{2}+\left(n_{s}+\tilde{n}\right)-G^{2} n_{0}^{2}
$$

and in the other $M-1$ mode

$$
v_{i}=\tilde{n}^{2}+\tilde{n} .
$$

The mean number of photons at the point of detection $z$ is

$$
\langle n\rangle=n_{s}+M \tilde{n}
$$

where $n_{s}=G \cdot n_{0}$. In a real receiver always more than just the signal mode is received. The number of signal modes to which the receiver is sensitive is $M=K B_{0} \Delta T$, where $B_{0}$ is the detector bandwidth and $\Delta T$ the timeconstant of the integrator in the detector [10], and $K=1$ if the detector is sensitive to one polarization and $K=2$ if the detector is sensitive to two polarizations. In agreement with [11], the variance of the detected signal is then from (A.8a) and (A.8b)

$$
V=\left(\sum_{M-1} v_{i}\right)+v_{0}
$$

assuming that the variance is equal over the total mode span defined by $M,($ A.10) takes the form

$$
V=M\left(\tilde{n}^{2}+\tilde{n}\right)+2 n_{s} \tilde{n}+n_{s}
$$

where it has been used that $n_{s}=G \cdot n_{0}$. The terms in (A.11) represent spontaneous-spontaneous beat noise, spontaneous shot noise, signal-spontaneous beat noise and signal shot noise. Due to the assumption of a coherent signal source the excess noise is not seen in (A.11).

In a digital signal represented by marks and spaces, where a space is achieved by emitting no photons within one bit period, the variance for a mark is given as in (A.11), whereas the variance in a space is given by

$$
V=M\left(\tilde{n}^{2}+\tilde{n}\right) .
$$

The electrical signal to noise ratio is defined as

$$
\frac{S}{N}=\frac{\langle n\rangle^{2}}{V}
$$

where $\langle n\rangle$ is the mean number of photons at the detector and $V$ the variance of the photons at the detector. Assuming that 
$2 n_{s} \tilde{n} \gg n_{s}+M \tilde{n}(\tilde{n}+1)$ the variance and mean, in presence of a mark, for the detected photons are

$$
\begin{aligned}
& V=2 n_{s} \tilde{n} \\
& n=n_{s}+M \tilde{n} .
\end{aligned}
$$

The above assumption that the signal-spontaneous noise power is dominating, is realistic for large signal powers as here where we are transmitting solitons.

In presence of a space the variance and mean of the detected photons are under the assumption $\tilde{n} \gg 1$

$$
\begin{aligned}
& V=M \tilde{n}^{2} \\
& n=M \tilde{n} .
\end{aligned}
$$

The detected number of photons are related to a detected energy through

$$
\begin{gathered}
E_{s}=n_{s} h \nu_{s} \\
\tilde{E}=n \tilde{h} \nu_{s}
\end{gathered}
$$

where (A.16a) represent the signal energy and (A.16b) the noise energy, $h \nu_{s}$ is the photon energy at the optical signal frequency $\nu_{s}$. Equation (A.16a) is valid for the signal energy propagating in the signal mode and (A.16b) is valid for the ASE energy propagating in the $M$ modes. In a digital signal the signal-spontaneous noise power is dominating when transmitting a mark, thus the variance and mean value of the received energy for a mark is

$$
\begin{aligned}
& \left\langle E(1)^{2}\right\rangle \sim 2 E_{s} \tilde{E} \\
& \langle E(1)\rangle=E_{s}+M \tilde{E} .
\end{aligned}
$$

Thus the electrical signal to noise ratio for a mark is

$$
\frac{S}{N}=\frac{E_{s}+2 M \tilde{E}}{2 \tilde{E}} \text {. }
$$

Introducing the signal energy in a mark relative to the noise energy through $S_{1}$

$$
S_{1}=\frac{E(1)}{\tilde{E}}
$$

(A.17a) and (A.17b) reduces to

$$
\begin{aligned}
& \left\langle S_{1}^{2}\right\rangle=\left\langle E(1)^{2}\right\rangle \frac{1}{\tilde{E}^{2}}=2 S_{1} \\
& \left\langle S_{1}\right\rangle=\langle E(1)\rangle \frac{1}{\tilde{E}}=S_{1}+M .
\end{aligned}
$$

For a space the variance and mean value of the received energy is

$$
\begin{aligned}
& \left\langle E(0)^{2}\right\rangle \sim M \tilde{E}^{2} \\
& \langle E(0)\rangle=M \tilde{E} .
\end{aligned}
$$

Introducing the signal energy in a space relative to the noise energy through $S_{0}$

$$
S_{0}=\frac{E(0)}{\tilde{E}}
$$

(A.21a) and (A.21b) reduces to

$$
\begin{aligned}
& \left\langle S_{0}^{2}\right\rangle=M \\
& \left\langle S_{0}\right\rangle=M .
\end{aligned}
$$

If the noise follows a Gaussian distribution the bit error rate is given by [13]

$$
\mathrm{BER}=\frac{1}{\sqrt{2 \pi}} \frac{\exp \left(-\frac{Q^{2}}{2}\right)}{Q}
$$

where the argument $Q$ to the error function is

$$
Q=\frac{\sqrt{\langle E(1)\rangle^{2}}-\sqrt{\langle E(0)\rangle^{2}}}{\sqrt{\left\langle E(1)^{2}\right\rangle}+\sqrt{\left\langle E(0)^{2}\right\rangle}}=\frac{\sqrt{\left(E_{s}+M \tilde{E}\right)^{2}}-M \tilde{E}}{\sqrt{2 E_{s} \tilde{E}}+\sqrt{M \tilde{E}^{2}}} .
$$

Which reduces to

$$
Q=\frac{S_{1}}{\sqrt{2 S_{1}}+\sqrt{M}}
$$

As emphasized previously $S_{1}$ is the signal energy relative to the noise energy in the beginning of an amplification period. The path average signal energy identical to the soliton energy relative to the signal energy in the beginning of an amplifier period is given by $R_{g}$ according to (6) in the main text. Thus when $\langle E\rangle$ is the path average signal energy $S_{1}$ is a function of $\alpha_{\mathrm{ASE}}$ and $R_{g}$ through the relation

$$
S_{1}=2 \frac{\langle E\rangle}{R_{g} \alpha_{\mathrm{ASE}} h \nu_{s} L_{\mathrm{tot}}}
$$

where $L_{\text {tot }}$ is the total transmission length. Equation (A.24) gives a bit error rate of $10^{-12}$ for $Q=7$. That is for $M=1$, the signal energy has to be more than 112 times the noise energy, whereas for $M=2$ the signal energy relative to the noise energy has to exceed 117 when a bit error rate lower than $10^{-12}$ is desirable.

\section{APPENDIX B}

Derivation of (13) For Calculation of Timing JitTER IN THE ARrival TIME OF A PULSE

The arrival time of a pulse is defined as the mean time of the pulse at the point of detection. Thus when the arrival time exhibits a stochastic behavior due to noise, both the average and variance of the pulse arrival time may be calculated. We consider an envelope pulse propagating in the fiber with the group velocity $v_{g}$. Changing to a retarded time variable $\left(t-z / v_{g}->t\right)$, the mean time $\langle t\rangle$ of an envelope pulse $\varphi(t)$ 
in this frame, moving with the group velocity, propagating from $z=0$ to $z$ is to a first-order approximation given as

$$
\left.\langle t\rangle\right|_{z}=\left.\langle t\rangle\right|_{z=0}+\left.\frac{\partial\langle t\rangle}{\partial z}\right|_{z=0} \cdot z
$$

where the first-order derivative of $\langle t\rangle$ with respect to the propagation coordinate $z$ is

$$
\begin{aligned}
i \frac{\partial}{\partial z}\langle t\rangle= & \frac{1}{\int \varphi \varphi^{*} d t}\left(\int \varphi^{*} t\left(i \frac{\partial \varphi}{\partial z}\right) d t\right. \\
& \left.+\int\left(-i \frac{\partial \varphi}{\partial t}\right)^{*} t \varphi d t\right) .
\end{aligned}
$$

In (B.2) and in the remaining of this appendix integration is performed from $-\infty$ to $+\infty$. In a nonlinear transmission line the governing equation for pulse propagation is the nonlinear Schrödinger equation [6]. Thus if $\beta_{\omega}^{\prime \prime}$ is the secondorder derivative of the propagation constant with respect to the angular frequency $\omega$ and $\Gamma$ includes the nonlinear Kerr coefficient, the Schrödinger equation is written as

$$
i \frac{\partial \varphi}{\partial z}=\left(\frac{1}{2} \beta_{\omega}^{\prime \prime} \frac{\partial^{2}}{\partial t^{2}}-\Gamma|\varphi|^{2}\right) \varphi .
$$

By insertion of (B.3) in (B.2) we achieve

$$
i \frac{\partial}{\partial z}\langle t\rangle=\frac{1}{\int \varphi \varphi^{*} d t}\left(\frac{1}{2} \beta_{\omega}^{\prime \prime} \int\left[\varphi \frac{\partial \varphi^{*}}{\partial t}-\varphi^{*} \frac{\partial \varphi}{\partial t}\right] d t\right) .
$$

Writing the pulse as an addition of a soliton $\varphi_{s}$ and a perturbating signal $\delta \varphi$, the mean time at $z$ given by (B.1) is rewritten as

$$
\begin{aligned}
\left.\langle t\rangle\right|_{z}= & \frac{1}{\int \varphi \varphi^{*} d t}\left(\int \varphi_{s}\left(t+\frac{1}{2} z \beta_{\omega}^{\prime \prime} i \frac{\partial}{\partial t}\right) \varphi_{s} d t\right. \\
& \left.+2 \operatorname{Real}\left[\int \delta \varphi^{*}\left(t+z \beta_{\omega}^{\prime \prime} i \frac{\partial}{\partial t}\right) \varphi_{s} d t\right]\right) .
\end{aligned}
$$

The first integral on the right-hand side represents the initial soliton mean time and frequency which equals zero. The second integral originates from the perturbating signal $\delta \varphi$ and is a scalar product of two functions $\delta \varphi$ and $\left(t+z \beta_{\omega}^{\prime \prime} i \partial / \partial t\right) \varphi_{s}$, respectively. When describing $\delta \varphi$ by an orthogonal set of functions the only contribution to the integral must satisfy the restriction in (B.6) given by

$$
\delta \varphi=c_{0}\left(t+z \beta_{\omega}^{\prime \prime} i \frac{\partial}{\partial t}\right) \varphi_{s}
$$

where $c_{0}$ is related to the noise energy in the signal mode. Other contributions from the orthogonal set of functions describing $\delta \varphi$ vanishes in the integral. The perturbation $\delta \varphi$ is related to the noise energy in the signal mode, $E_{\mathrm{ASE}}$ by

$$
\int \delta \varphi^{*} \delta \varphi d t=E_{\mathrm{ASE}}=\left|c_{0}\right|^{2} B
$$

Where $B$ is given by

$$
\begin{aligned}
B=\int( & t^{2}\left|\varphi_{s}\right|^{2}+\left(z \beta_{\omega}^{\prime \prime}\right)^{2} \frac{\partial \varphi_{s}}{\partial t} \frac{\partial \varphi_{s}^{*}}{\partial t}+t \varphi_{s} z \beta_{u}^{\prime \prime} i \frac{\partial \varphi_{s}^{*}}{\partial t} \\
& \left.-t z \beta_{\omega}^{\prime \prime} i \frac{\partial \varphi_{s}}{\partial t} \varphi_{s}^{*}\right) d t
\end{aligned}
$$

With $c_{0}=e^{i \alpha}\left(E_{\mathrm{ASE}} / B\right)^{1 / 2}$ from (B.7) $\delta t$ is found by inserting (B.6) in (B.5). This gives $\delta t$ the following form:

$$
\delta t=\frac{2}{\int \varphi \varphi^{*} d t} \sqrt{E_{\mathrm{ASE}}} \sqrt{B} \cos (\alpha) .
$$

In (B.8) we recognize the initial angular frequency and time root mean square (RMS) pulse widths $\omega_{\mathrm{RMS}}$ and $T_{\mathrm{RMS}}$ in the terms

$$
\begin{aligned}
-\int \varphi_{s} \frac{\partial^{2} \varphi_{s}^{*}}{\partial t^{2}} d t & =\omega_{\mathrm{RMS}}^{2} \cdot \int \varphi \varphi_{s}^{*} d t \\
\int t^{2}\left|\varphi_{s}\right| d t & =T_{\mathrm{RMS}}^{2} \cdot \int \varphi \varphi_{s}^{*} d t .
\end{aligned}
$$

The average value of (B.9) equals zero whereas the variance of (B.9) is the Gordon-Haus variance, written as

$$
\begin{aligned}
\left\langle\delta t^{2}\right\rangle= & 2 \frac{\int|\delta \varphi|^{2} d t}{\int\left|\varphi_{s}\right|^{2} d t}\left(T_{\mathrm{RMS}}^{2}+\left(z \beta_{\omega}^{\prime \prime}\right)^{2} \omega_{\mathrm{RMS}}^{2}\right. \\
& \left.+\frac{2}{\int \varphi \varphi_{s}^{*} d t} \beta_{\omega}^{\prime \prime} z \operatorname{Im}\left[\int \frac{\partial \varphi_{s}}{\partial t} t \varphi_{s}^{*} d t\right]\right) .
\end{aligned}
$$

The last term in (B.11) is recognized as chirp in the pulse. In (B.11) both the mean noise power $P_{\mathrm{ASE}}$, resulting from amplified spontaneous emission (ASE) and mean signal power $P_{\text {SIG }}$ after an amplifier occurs through

$$
\frac{\int|\delta \varphi|^{2} d t}{\int\left|\varphi_{s}\right|^{2} d t}=\frac{E_{\mathrm{ASE}}}{E_{\mathrm{SIG}}}=\frac{P_{\mathrm{ASE}}}{P_{\mathrm{SIG}}}
$$

where $E_{\mathrm{ASE}}$ and $E_{\mathrm{SIG}}$ is the noise and signal energy in the signal mode. Thus the variance in the arrival time given by (B.11) is minimized by maximizing the signal power and minimizing the noise power. Equation (B.11) is valid for any pulse shape and therefore more general than the normal Gordon-Haus variance [8]. In long-distance transmission systems the term originating from $\omega_{\mathrm{RMS}}^{2}$ in (B.11) is dominating due to the $z^{2}$ dependency. Thus (B.11) approximates to

$$
\left\langle\delta t^{2}\right\rangle=2 \frac{E_{\mathrm{ASE}}}{E_{\mathrm{SIG}}}\left(z \beta_{\omega}^{\prime \prime}\right)^{2} \omega_{\mathrm{RMS}}^{2}
$$

For solitary pulses with $\tau$ as the pulse width at half maximum intensity, the mean signal energy $E_{\mathrm{SIG}}$ after an amplifier and the RMS angular frequency pulse width $\omega_{\mathrm{RMS}}$ is expressed as

$$
\begin{array}{r}
E_{\mathrm{SIG}}=\frac{P_{\mathrm{sol}} \cdot \tau}{\ln (\sqrt{2}+1)} \frac{1}{R_{g}} \\
\omega_{\mathrm{RMS}}^{2}=\frac{1}{3}\left(\frac{2}{\tau} \ln (\sqrt{2}+1)\right)^{2}
\end{array}
$$

where $P_{\text {sol }}$ is the peak power of a first-order soliton and $R_{g}$ is the factor that relates the path average signal power to the signal power launched to the transmission line. Thus the total variance in the arrival time after $N$ amplifiers is achieved by adding the contribution from each amplifier located at position $z_{n}=n z_{a}$ where $z_{a}$ is the separation between two amplifiers, 
here in case of distributed amplification the amplification period, each with gain $G$ and noise figure $F$. This gives

$$
\begin{aligned}
\left\langle\delta t^{2}\right\rangle= & 2 \frac{\tilde{n} h \nu_{s}}{P_{\mathrm{sol}} \tau} \ln (\sqrt{2}+1) R_{g}\left(\frac{D \lambda^{2}}{2 \pi c}\right)^{2} \frac{4}{3 \tau^{2}} \\
& \cdot(\ln (\sqrt{2}+1))^{2} \sum_{N}\left(L_{\mathrm{tot}}-z_{n}\right)^{2}
\end{aligned}
$$

where $\tilde{n}$ is the number of ASE noise photons propagating in the signal mode, $h \nu_{s}$ is the photon energy at the signal wavelength $\lambda, \mathrm{D}$ is the fiber dispersion, $L_{\text {tot }}$ is the total transmission length, and $c$ is the velocity of light. Approximating the summation with an integration over the total transmission length as in [8] we achieve

$$
\left\langle\delta t^{2}\right\rangle=\frac{2 \ln (\sqrt{2}+1)}{9} h \frac{D L_{\text {tot }}^{3} n_{k e}}{\tau A_{\text {eff }}} \frac{\alpha_{\mathrm{ASE}}}{2} R_{g}
$$

where $\alpha_{\mathrm{ASE}}$ is $(F G-1) /\left(z_{a}\right), n_{k e}$ the nonlinear Kerr coefficient and $A_{\text {eff }}$ the effective fiber core area defined by (1) in the main text.

\section{ACKNOWLEDGMENT}

The authors would like to thank Dr. E. Nicolaisen for fruitful discussions and suggestions.

\section{REFERENCES}

[1] T. J. Whitley, C. A. Millar, S. P. Craig-Ryan, and Paul Urquhart, "Demonstration of a distributed optical fibre amplifier BUS network," in Proc. Opt. Amplifiers Their Appl., 1990, pp. 236-237.

[2] D. L. Williams, S. T. Davey, M. Spirit, G. R. Walker, and B. L. Ainslie "Transmission and saturation performance of dispersion-shifted distributed erbium fibre," Electron. Lett., vol. 27, no. 10, pp. 812-813, 1991.

[3] B. Pedersen, A. Bjarklev, and J. H. Povlsen, "Design of erbium doped fibre amplifiers for $980 \mathrm{~nm}$ or $1480 \mathrm{~nm}$ pumping," Electron. Lett. vol. 27 , no. 3, pp. $255-256,1991$.

[4] K. Dybdal, N. Bjerre, J. E. Petersen, and C. C. Larsen, "Spectroscopic properties of Er-doped silica fibers and preforms," in Proc. SPIE'89, 1989, pp. $209-218$.

[5] K. Rottwitt, J. H. Povlsen, and A. Bjarklev, "Demand for accuracy of the attenuation constant in distributed active fibers," in Proc. SPIE'9I OE/FIBERS, 1991, paper 1581-20.

[6] L. F. Mollenauer, S. G. Evangelides Jr., and H. A. Haus, "Long-distance propagation using lumped amplifiers and dispersion shifted fiber," $J$. Lightwave Technol., vol. 9, no. 2, pp. 194-197, 1991.

[7] P. L. Danielsen, "Analytical expressions for group delay in the far field from an optical fibre having an arbitrary index profile," $J$. Quantum Electron., vol. 19 , pp. $850-853,1981$.

[8] J.P. Gordon and H.A. Haus, "Random walk of coherently amplified solitons in optical fiber transmission," Opt. Lett., vol. 11, no. 10, pp. $665-667,1986$

[9] R. H. Stolen and C. Lin, "Self-phase-modulation in silica optical fibers," Phys. Rew. A., vol. 17, no. 4, pp. 1448-1453, 1978.

[10] J.P. Gordon and L.F. Mollenauer, "Effects of fiber nonlinearities and amplifier spacing on ultra-long transmission," J. Lightwave Technol. vol. 9 , no. 2 , pp. $170-173,1991$

[11] K. Shimoda, H. Takahasi, and C.H. Townes, "fluctuations in amplification of quanta with application to maser amplifiers," J. Physical Soc. Japan, vol. 12, no. 6, pp. $686-700,1957$.
[12] B. Pedersen, A. Bjarklev, J. H. Povlsen, K. Dybdal, and C. C. Larsen, "The design of erbium-doped fiber amplifiers," J. Lightwave Technol. vol. 9, pp. 1105-1112, 1991

[13] N. A. Olsson, "Lightwave systems with optical amplifiers," J. Lightwave Technol., vol. 7, no. 7, pp. 1071-1082, 1989.

[14] L. F. Mollenauer, J. P. Gordon, and M. N. Islam, "Soliton propagation in long fibers with periodically compensated loss," J. Quantum Electron. vol. QE-22, no. 1, pp. $157-173,1986$.

[15] K. Rottwitt, et al., "Detailed analysis of distributed erbium doped fibre amplifiers," accepted for presentation at Optical Amplifiers and their Application July 1992.

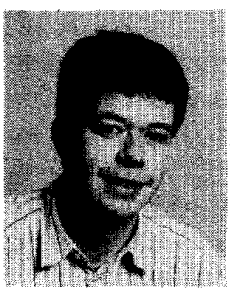

Karsten Rottwitt was born in Odense, Denmark, on November 13, 1965. He received the M.Sc. degree in electrical engineering in 1990 from the Electromagnetics Institute, Technical University of Denmark. He is presently working toward the Ph.D. degree at the Electromagnetics Institute on soliton communication systems.

Anders Bjarklev, photograph and biography not available at the time of publication.

Jørn Hedegaard Povlsen, photograph and biography not available at the time of publication.

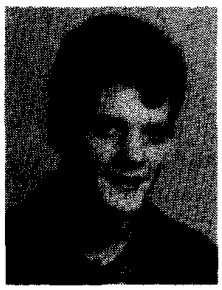

Ole Lumholt was born in Copenhagen, Denmark, on November 20, 1963. He received the M.Sc. degree in electrical engineering in 1990 from the Electromagnetics Institute, Technical University of Denmark.

He is presently working at the Center for Broadband Telecommunications, Electromagnetics Institute, on active optical waveguides.

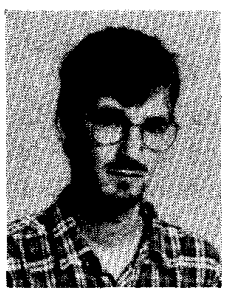

Thomas P. Rasmussen was born in Copenhagen, Denmark, on November 3, 1965. He received the M.Sc. degree in electrical engineering in 1991 from the Electromagnetics Institute, Technical University of Denmark.

$\mathrm{He}$ is presently working at the Center for Broadband Telecommunications, Electromagnetics Institute on active optical waveguides. 